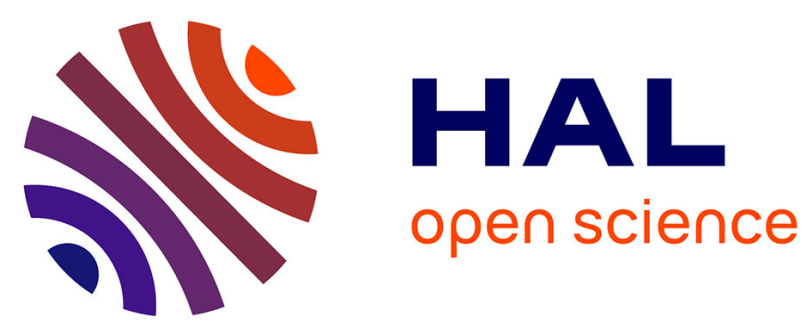

\title{
Force measurements on rising bubbles
}

Woodrow L. Shew, Sébastien Poncet, Jean-Francois Pinton

\section{To cite this version:}

Woodrow L. Shew, Sébastien Poncet, Jean-Francois Pinton. Force measurements on rising bubbles. 2006. hal-00013378v2

\section{HAL Id: hal-00013378 https://hal.science/hal-00013378v2}

Preprint submitted on 16 May 2006

HAL is a multi-disciplinary open access archive for the deposit and dissemination of scientific research documents, whether they are published or not. The documents may come from teaching and research institutions in France or abroad, or from public or private research centers.
L'archive ouverte pluridisciplinaire HAL, est destinée au dépôt et à la diffusion de documents scientifiques de niveau recherche, publiés ou non, émanant des établissements d'enseignement et de recherche français ou étrangers, des laboratoires publics ou privés. 


\title{
Force measurements on rising bubbles
}

\author{
Woodrow L. Shew, Sebastien Poncet, and Jean-François Pinton \\ Laboratoire de Physique, École Normale Supérieure de Lyon \\ Lyon, France 69007
}

\begin{abstract}
The dynamics of millimeter sized air bubbles rising through still water are investigated using precise ultrasound velocity measurements combined with high speed video. From measurements of speed and three dimensional trajectories we deduce the forces on the bubble which give rise to planar zigzag and spiraling motion.
\end{abstract}

\section{BACKGROUND}

An understanding of bubble-fluid interactions is important in a broad range of natural, engineering, and medical settings. Air-sea gas transfer, bubble column reactors, oil/natural gas transport, boiling heat transfer, ship hydrodynamics, ink-jet printing and medical ultrasound imaging are just a few examples where the dynamics of bubbles play a role (e.g $[6,16,26])$.

We narrow our focus to a single air bubble rising through still water. The buoyant force which drives the bubble's rise does work resulting in an increase in kinetic energy of the surrounding fluid. This induced flow, in turn, gives rise to hydrodynamic forces on the bubble and hence changes in the bubble trajectory. The measurement of these forces is the aim of the experimental work presented here

Our study includes a range of bubble sizes between 0.84 and $1.2 \mathrm{~mm}$ in radius. At the small end of this range the bubble's path is rectilinear. As the bubble size is increased, one observes a transition to a planar zigzag path $[8,23]$. A second instability, often preceded by the zigzag, results in a spiraling path $[5,8,15,23]$. For even larger bubbles, a third type of oscillating path occurs, which has similarities to the zigzag, sometimes called "rocking". We do not address this state and emphasize that it is different than the zigzag mentioned above. Unlike the zigzag state that we study, the rocking bubble undergoes dramatic shape oscillations and the frequency of path oscillation is several times higher than the zigzag or spiral $[5,15]$. Our approach is to use well resolved measurements of three-dimensional bubble trajectories to calculate the hydrodynamic forces on the bubbles.

The dynamics of bubble path instabilities have puzzled researchers for quite a long time. Leonardo Da Vinci is likely the first scientist to have contributed to the significant body of work addressing this problem [26]. Clift, Grace, and Weber [6] review relevant studies prior to about 1978. In 2000, Magnaudet and Eames [16] provided a thorough account of more recent work on this subject. Our attention will be limited to those works which address path instabilities of bubbles less than 2.5 $\mathrm{mm}$ in diameter. Saffman [27], Hartunian and Sears [11], and later Benjamin [4] attempted to explain features of the path instabilities and bubble shape by analytical means, but experiments are not in accord with their findings.
Several experimental works have visualized and documented zigzagging and spiraling bubble paths. Aybers and Tapucu [1, 2] used photographic techniques to measure bubble speed, drag coefficients, size, shape, and path. Mercier, Lyrio, and Forslund [17] used a stroboscope and several cameras to measure short sections of bubble trajectories. More recently, Wu and Gharib used a high speed video three-dimensional imaging system to measure paths and bubble shape. These works advanced qualitative understanding of bubble behavior without attempting to explain causal mechanisms or make detailed quantitative force measurements.

Other recent studies have investigated path instabilities with special attention paid to the role of the bubble's wake. Lunde and Perkins [15] used dye to observe the wake of ascending bubbles and solid particles. Brücker [5] used particle image velocimetry to study the wake of large spiraling and rocking bubbles. Mougin and Magnaudet [22, 23] presented numerical observations of the path and wake of a bubble with a rigid ellipsoidal shape. de Vries et al. [8] used Schlieren optics techniques to visualize the wakes of zigzagging and spiraling bubbles. Finally, Ellingsen and Risso [10] used laser Doppler anenometry and cameras to measure the path as well as the flow around the bubble.

These studies have revealed a wake consisting of two long, thin, parallel vortices aligned with the bubble's path. One vortex rotates clockwise and the other counter-clockwise. For a spiraling bubble the wake vortices are continuously generated, while they are interrupted twice per period of path oscillation for the zigzag. Mougin and Magnaudet [22, 23] observed a nearly identical wake structure in their numerical simulations (see also $[24])$. It is believed that the wake vortices play a critical role in generating hydrodynamic forces on the bubble.

In the next section we describe the experimental apparatus and measurement techniques, as well a typical bubble trajectory. In section III, we present a method for extracting force measurements from path and velocity measurements based on the generalized Kirchhoff equations. Then, in section IV, we discuss some observations of the bubble trajectory during the first few hundred milliseconds after release. We describe observations and force measurements for zigzagging bubbles in section $\mathrm{V}$ and spiraling bubbles in section VI. Finally, our results are summarized in section VII. 


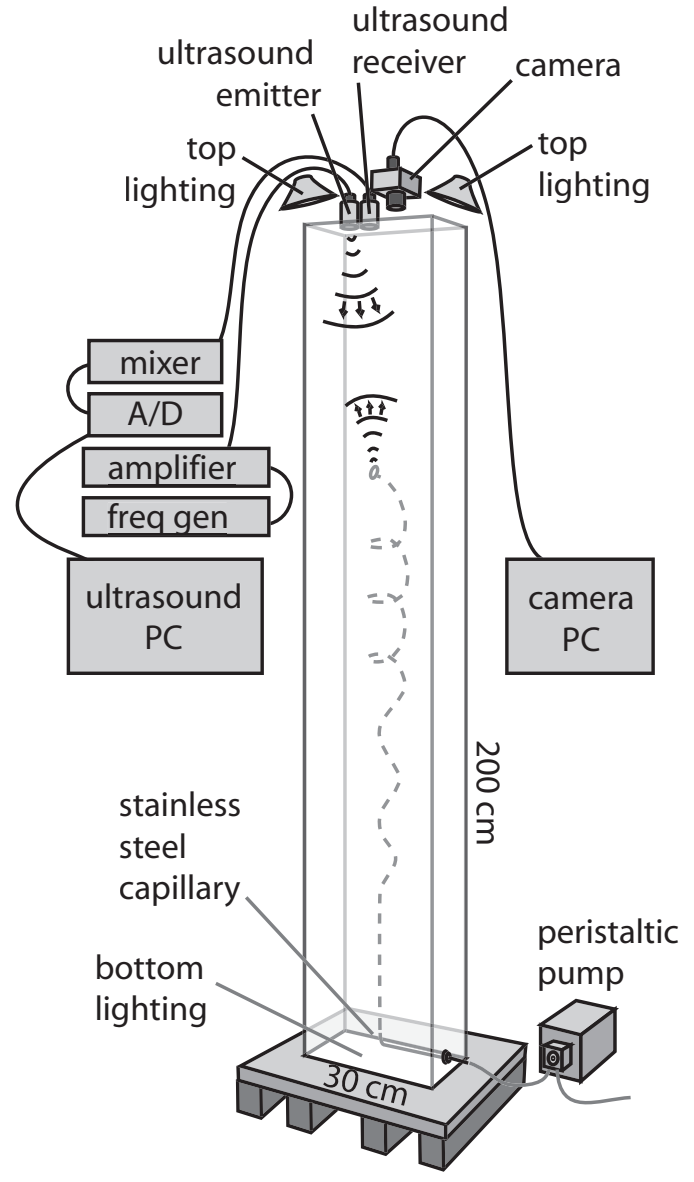

FIG. 1: Schematic of experimental setup. As the bubble rises its vertical velocity is measured using ultrasound and its horizontal position is obtained with a high speed video camera.

\section{EXPERIMENTAL APPARATUS AND METHODS}

One goal of this work is to obtain measurements of bubble behavior rising through a large distance, revealing the long time dynamics of the zigzag and spiral instabilities. To this effect, the experiments are conducted in a tank $2 \mathrm{~m}$ in height and $30 \mathrm{~cm}$ wide with square cross section as illustrated in Fig. 1. The walls are made of $1.45 \mathrm{~cm}$ thick acrylic plate. Bubbles are produced at the bottom of the vessel by pumping air through a 24 gauge stainless steel capillary tube with a $0.30 \mathrm{~mm}$ inner diameter (ID) and a $0.56 \mathrm{~mm}$ outer diameter (OD). The tube is oriented with its open end facing upwards. The air is pumped to the capillary tube through a length of Tygon tubing (0.51 mm ID and $2.3 \mathrm{~mm}$ OD, from ColeParmer) using a peristaltic pump (Roto-Consulta, flocon 1003). The rotor of the pump is turned by hand, releasing a single bubble. We always allow at least 3 minutes delay between the release of consecutive bubbles to be sure that the water is truly quiescent for each bubble.

The volume of each bubble is measured individually. When the bubble reaches the top of the vessel it is

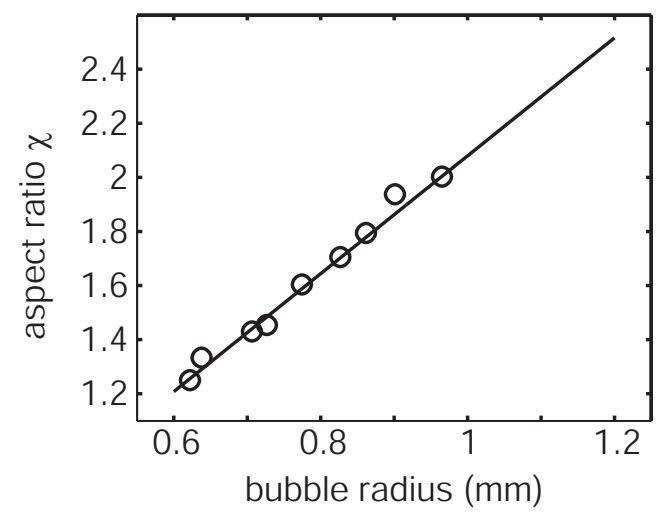

FIG. 2: Comparison of Duineveld's [9] measurements of aspect ratio and the linear model we use to estimate $\chi$.

trapped under a submerged plate. Using a syringe, each bubble is sucked into a transparent section of Tygon tubing (ID $0.51 \pm 0.005 \mathrm{~mm}$ ) with water on either side of the bubble. The length of the air plug in the tube is then measured with calipers ( $\pm 0.2 \mathrm{~mm}$ precision). Knowing the tube inner diameter one then calculates the bubble volume. In the results that follow, an equivalent radius $R \equiv(3 / 4 \pi \times \text { actual volume })^{1 / 3}$ is used as a measure of the bubble size. During the ascent, $R$ increases by $6 \%$ due to the gradient in hydrostatic pressure. This expansion is accounted for in the calculations of forces. Furthermore, each instance where the bubble radius or Reynolds number is presented in this paper it is properly adjusted for the pressure at the height of the bubble being described. The Reynolds number is defined $R e=2 R U / \nu$, where $U$ is the current speed of the bubble and $\nu$ is the kinematic viscosity of water. We have observed speeds in the range $32<U<36 \mathrm{~cm} / \mathrm{s}$, yielding Reynolds numbers $500<R e<770$.

Some of our force calculations depend on the shape of the bubble. It has been shown by other experimental studies that the bubble is close to an oblate ellipsoid $[7,9,10]$. Since we do not make such measurements, we use the experimental results of Duineveld [9] to estimate the shape of our bubbles. Wu and Gharib [30] measured aspect ratios which confirm Duineveld's results. The aspect ratio of the bubbles in our size range is well approximated by a linear function of bubble equivalent radius. The aspect ratio $\chi$ is defined as the length of the semimajor axis divided by the length of the semiminor axis. In fig. 2, we show Duineveld's results and the linear fit,

$$
\chi(R)=2.18 R-0.10 .
$$

This method of estimating $\chi$ is supported by the agreement of our measurements with Moore's [18] drag theory as shown below in Fig. 7, section IV.

Before each experiment the vessel and all parts exposed to the water are thoroughly cleaned with methanol, dried, and then rinsed with tap water for 5 minutes. All data is collected with tap water less than 8 hours old. It is 
known that small bubbles rise more slowly in tap water compared to highly purified water due to contamination of the air-water interface with surfactants (e.g. $[6,9])$. Nonetheless, several observations suggest that surfactant effects are not greatly influencing the dynamics of our bubbles, probably because of their larger size. First, our velocity measurements are consistent with Moore's drag theory and Duineveld's measurements in clean water (see fig. 7 in section IV). Second, we observe that during the straight rise of a bubble of radius $1.0 \mathrm{~mm}$ (at 1 atm), the velocity grows by about $2 \%$ over $1.5 \mathrm{~m}$. This result is consistent with the increase in buoyancy and drag due the expansion of size as well as aspect ratio during ascent. If surfactant effects were significant, the bubble would likely slow down as it rises. We note that for bubbles smaller than about $0.75 \mathrm{~mm}$ in radius, our measured rise velocities reveal such a decrease in speed and are lower than those reported by Duineveld. This indicates that, in our tap water, smaller bubbles are strongly influenced by surfactants, while larger bubbles are not. The data presented in this paper is limited to bubbles larger than $0.87 \mathrm{~mm}$

Temperature is monitored at two different depths for each experiment. The mean temperature is $18.5 \pm 0.25^{\circ} \mathrm{C}$ and the temperature gradient is always less than 0.009 ${ }^{\circ} \mathrm{C} / \mathrm{cm}$.

The trajectory of the bubble is measured using two methods: ultrasound and high speed video. The vertical component of the bubble velocity is obtained with high precision using a continuous ultrasound technique. We briefly describe this technique here, but for more detail the reader is referred to [19-21]. One piezo-electric ultrasound transducer positioned at the top of the vessel generates a continuous $2.8 \mathrm{MHz}$ sound wave directed towards the bottom. The emitted waveform is created by a Agilent arbitrary function generator (E1445A) and amplified by a Kalmus Engineering Model 150C 47 dB radio frequency power amp. The sound is scattered by the rising bubble and measured by an array of eight piezo ultrasound transducers (custom by Vermon), also located at the top of the vessel. Each signal measured by the eight receiving transducers is then mixed into a different frequency band, amplified, and summed using an in-house custom made circuit. After this stage, $130 \mathrm{~dB}$ of dynamical range is preserved. The eight channels are mixed down to one channel so that only one analog to digital converter is needed to digitize the data. The digitization is accomplished with a Agilent $10 \mathrm{MHz}, 23 \mathrm{bit} \mathrm{A} / \mathrm{D}$ converter (E1430A). All of the Agilent devices are VXI modules in a Agilent mainframe (E1421A) which interfaces with a personal computer using a fire-wire module (E8491A).

Once digitized, Matlab routines are used to extract the original eight channels of ultrasound data. The signal from each channel is dominated by the emitted frequency $(2.8 \mathrm{MHz})$ and a lower amplitude Doppler shifted frequency of the sound scattered from the bubble. Each signal is shifted in frequency so that the emitted frequency becomes DC and hence the Doppler shifted frequency is directly proportional to the bubble velocity. In order to obtain velocity as a function of time the frequency is extracted using a numerical approximated maximum likelihood scheme coupled with a generalized Kalman filter [19].

The ultrasound method measures the component of the bubble's velocity along the line between the bubble and the ultrasound receiver. In order to obtain the true vertical component, some correction of the data is required as the bubble comes closer to the ultrasound transducer. An iterative corrective algorithm is employed beginning with the average vertical velocity and the position data from the camera data as a first estimate of the trajectory. Geometrical corrections are then made on the original velocity data based on this approximate trajectory. This new corrected velocity is then used to recompute the trajectory. The process is then iterated until convergence is reached.

The absolute accuracy of our velocity measurements was verified using a video camera. We released an object slightly heavier than water and recorded its steady descent with the ultrasound device and simultaneously with a camera positioned 2.5 meters away with a side view of the tank. The test showed that the ultrasound velocity measurements are consistent within $2 \%$ of the camera data. We measure top speeds of our bubbles typically about $36 \mathrm{~cm} / \mathrm{s}$, which is consistent with other experimental measurements $[2,9,30]$. The relative accuracy of our velocity measurements is more precise, typically $\pm 1 \mathrm{~mm} / \mathrm{s}$, or about $0.2 \%$ accuracy. Furthermore, the sampling frequency is several $\mathrm{kHz}$. Over a distance of 2 meters, this level of accuracy is not possible with cameras or other optical methods. Another advantage is that the ultrasound technique is potentially useful in opaque fluids.

The vertical velocity measurements provide direct observations of the kinetic energy delivered to the fluid as the bubble rises. The only energy source in the system is the work done by the buoyancy force $\mathbf{F}_{b}$. The power delivered to the fluid is then $\mathbf{F}_{b} \cdot \mathbf{U}=\rho V g U_{z}$, where $\rho=\rho_{f}-\rho_{g}$ is the density difference between the fluid and the gas, $V$ is the volume of the bubble, $g$ is acceleration due to gravity, and $U_{z}$ is the vertical component of velocity of the bubble. Note that $\rho_{g} \ll \rho_{f}$, so $\rho \approx \rho_{f}$. In the remainder of this paper we will continue to neglect the density of the gas. For typical bubbles in our study, $U_{z} \approx 0.35 \mathrm{~m} / \mathrm{s}$ and the buoyancy force is $\rho V g \approx 50 \mu \mathrm{N}$. Therefore the bubble produces about $10 \mu \mathrm{W}$ of power in the form of fluid kinetic energy as it rises.

A high speed video camera (Photron Fast Cam Ultima 1024) is used to obtain the horizontal motion of the bubble. The camera is positioned above the vessel close to the ultrasound receiving array so that it records the lateral movement of the bubble. The camera is operated at 125 frames/sec with $512 \times 512$ pixel resolution. The bubble is between 10 and 100 pixels wide as it rises from a depth of two meters. Lighting is provided by two in- 
candescent lamps (125 and 100 Watts) positioned above the vessel and one (100 Watts) beneath the translucent floor. Note that most of the time series displayed later in this paper contain 125 samples/sec since they are at least partially derived from the camera data. This gives an effective time resolution of $8 \mathrm{~ms}$. Time series with higher sampling rates (see section IV) are derived only from the ultrasound data, which yields a time resolution of less than $1 \mathrm{~ms}$. The period of motion for typical path oscillations is around $200 \mathrm{~ms}$.

The bubble position is extracted from movies using another Matlab routine. The routine subtracts frame $i$ from frame $i+1$, then averages over a $5 \times 5$ pixel moving window and locates the maximum of the resulting image. This process is repeated, reversing the subtraction (frame $i$ minus frame $i+1$ ). The position of the maxima of the two subtraction/averageing processes are then averaged and taken as the bubble position. This method is found to reliably locate the bubble center even when the camera focus and light reflected from the bubble changes during its ascent. The accuracy of the position measurements is about $3 \%$ or $\pm 0.1 \mathrm{~mm}$. The horizontal position data is differentiated to obtain the horizontal velocity with about $6 \%$ or $\pm 6 \mathrm{~mm} / \mathrm{s}$ precision.

Trajectories were recorded for over 20 bubbles. From the vertical speed and horizontal position data we may reconstruct the entire three dimensional trajectory for each bubble as demonstrated in Fig. 3. The bubble in Fig. 3 is $1.12 \mathrm{~mm}$ in radius at atmospheric pressure. This example clearly demonstrates the three different types of behavior exhibited by the bubbles in the size range of our investigation. Just after the bubble is generated it accelerates quickly to its terminal speed. It rises for a short time in a nearly straight path. For a large enough bubble, the rectilinear rise soon becomes unstable to a zigzag motion. These oscillations are confined to a vertical plane (y-z plane in Fig. 3). The path then evolves into a spiral. A smooth transition occurs from zigzag to elliptical spiral, and finally to a circular spiral. This transition is shown in Fig. 4, where the trajectory is projected onto a horizontal plane.

\section{FORCES ON BUBBLES}

The equations of motion for a rigid body moving through a fluid at rest were established in the context of potential flow theory more than a century ago by Kirchhoff (see chapter VI in [13]). Like in other analytical approaches to understanding bubble dynamics, potential flow theory describes the gross features, but regions of the flow with vorticity must be accounted for in order to make precise predictions. Kirchhoff's equations have been generalized to the case of viscous, rotational flow [12] and, more recently, used in numerical work [22, 23] to investigate the behavior of freely rising bubbles with a fixed shape. The numerical work revealed the same zigzagging and spiraling paths as we and others have ob- served experimentally as well as quantitative agreement with path oscillation amplitudes and frequencies. These results strongly suggest that shape changes to the bubble do not play a critical role in the dynamics. Based on this result and experimental observations [10] of steady bubble shapes for the size range we study, we assume that bubble shape is fixed and use the generalized Kirchhoff equations. (We note that de Vries et al. [8] report that zigzagging bubbles may have a slightly oscillating shape. We will address the consequences of this possibility for our measurement uncertainty later in section V.)

\section{A. Equations of motion}

The Kirchhoff equations govern the six degrees of freedom necessary to completely specify the angular velocity $\boldsymbol{\Omega}$ and the linear velocity $\mathbf{U}$ of a body (eqns. 8 and 7 below). Although the Kirchhoff equations are well established, we will revisit the main features of the derivation of the equation for velocity $\mathbf{U}$ in order to clarify the nature of the equations and emphasize the proper use of reference frames and coordinate systems.

First, recall that with an expression for the kinetic energy $\mathcal{T}$ and potential energy $\mathcal{U}$ of the entire system in terms of only the bubble motion, one may derive equations of motion for the bubble using Lagrange's formalism. The system lagrangian is $\mathcal{L}=\mathcal{T}-\mathcal{U}$ and the equations of motion are

$$
\frac{d}{d t}\left(\frac{\partial \mathcal{L}}{\partial U_{n}^{L}}\right)-\frac{\partial \mathcal{L}}{\partial X_{n}^{L}}=F_{n}^{L} .
$$

where the superscript $L$ indicates that the variables are expressed in a lab-fixed (Galilean) coordinate system, $\mathbf{X}^{L}$ is the bubble position, and $\mathbf{F}^{L}$ are the forces acting on the bubble which are not expressed in the potential energy term. The potential energy is simply $\mathcal{U}=-\rho V g z$. The kinetic energy is $\mathcal{T}=\mathcal{T}_{\text {liquid }}+\mathcal{T}_{\text {bubble }} \approx \mathcal{T}_{\text {liquid }}$, since the bubble's mass is much smaller than that of the fluid it sets in motion. If the bubble is far from the boundaries of the fluid domain, $\mathcal{T}$ is generally a quadratic function depending only on $\boldsymbol{\Omega}$ and $\mathbf{U}$ (see [13] for potential flow case and [12] for more general treatment). For an ellipsoidal body like our bubbles,

$$
\mathcal{T}=U_{i}^{L} \mathbb{A}_{i j}^{L} U_{j}^{L}+\Omega_{i}^{L} \mathbb{D}_{i j}^{L} \Omega_{j}^{L}
$$

where $\mathbb{A}^{L}$ and $\mathbb{D}^{L}$ are called the added mass tensor and added rotational inertia tensors. If the bubble's motion is rectilinear, then $\mathbb{A}^{L}$ and $\mathbb{D}^{L}$ are constant in time, depending only on the bubble shape. When the bubble orientation changes with time, $\mathbb{A}^{L}$ and $\mathbb{D}^{L}$ become time dependent as well. This point is clarified when $\mathbb{A}_{i j}^{L} U_{j}^{L}$ is interpreted as the linear momentum imparted to the fluid in the $i$ direction due to the motion of the bubble in the $j$ direction. The equations of motion are then

$$
\frac{d}{d t}\left(\mathbb{A}_{m n}^{L} U_{n}^{L}\right)=F_{n}^{L}+F_{B n}^{L},
$$



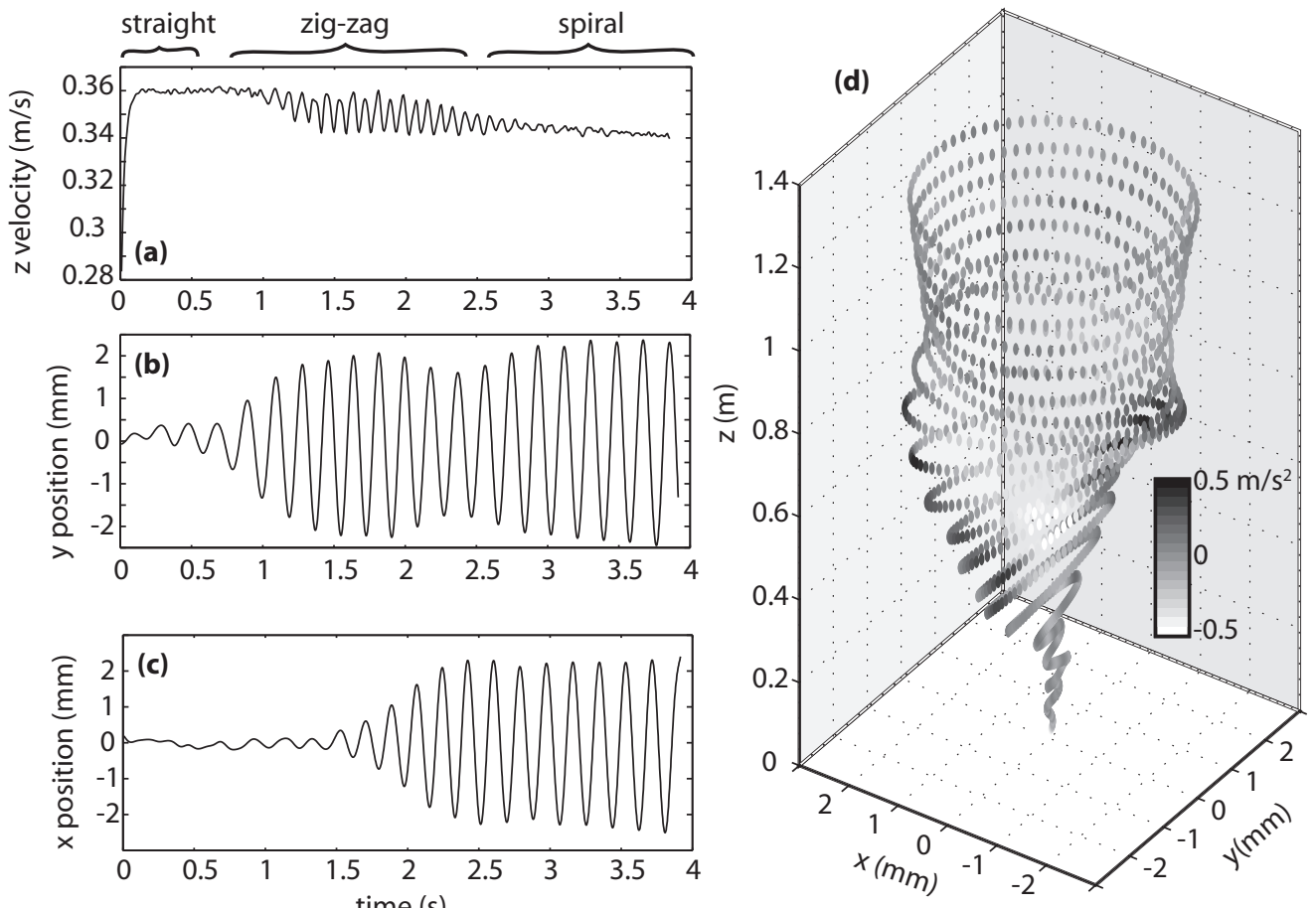

FIG. 3: Example trajectory of a $1.12 \mathrm{~mm}$ radius bubble (at $1 \mathrm{~atm}$ ). (a) Vertical component of velocity as measured with ultrasound technique, (b) y position from camera data, (c) $x$ position from camera data, and (d) three dimensional reconstruction of full trajectory with grayscale indicating magnitude of acceleration. The bubble begins rising straight, followed by zigzag motion in the $(y, z)$ plane with oscillating velcocity, followed by a three-dimensional spiral motion with steady velocity.

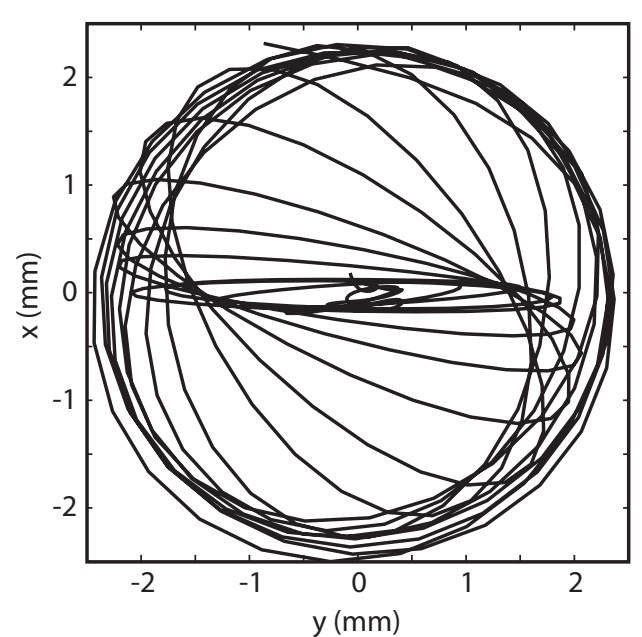

FIG. 4: Projection of a bubble trajectory onto a horizontal plane during the transition from zigzag to spiral. The bubble radius is $1.12 \mathrm{~mm}$ at $1 \mathrm{~atm}$. The time step between plotted points is $8 \mathrm{~ms}$.

where $\mathbf{F}_{B}^{L}=(0,0, \rho V g)$ is the buoyancy force resulting from the potential energy term.

It is convenient to recast this equation in terms of quantities which are projected onto a bubble-fixed coordinate system, for example $U_{i}=\mathbb{R}_{i j} U_{j}^{L}$. The components of the orthogonal projection operator $\mathbb{R}$ are the direction cosines, which define the orientation of the bubble with respect the the lab-fixed coordinates. Then equation 4 becomes,

$$
\frac{d}{d t}\left(\mathbb{R}_{m l}^{-1} \mathbb{A}_{l j} \mathbb{R}_{j k} \mathbb{R}_{k n}^{-1} U_{n}\right)=\mathbb{R}_{m n}^{-1} F_{n}+\mathbb{R}_{m n}^{-1} F_{B n},
$$

where $\mathbb{A}$ is now time independent. Using the fact that the time derivative of $\mathbb{R}_{i j}$ is $\mathbb{R}_{r j} \epsilon_{i r s} \Omega_{s}$, where $\epsilon$ is the permutation tensor, the resulting equation is

$$
\mathbb{R}_{m l}^{-1} \mathbb{A}_{l j} \frac{d U_{j}}{d t}+\mathbb{A}_{l j} U_{j} \mathbb{R}_{m r}^{-1} \epsilon_{r s l} \Omega_{s}=\mathbb{R}_{m n}^{-1} F_{n}+\mathbb{R}_{m n}^{-1} F_{B n} .
$$

Multiplying this result by $\mathbb{R}_{i m}$ and relabelling some indices, we have Kirchhoff's equation for the bubble velocity,

$$
\mathbb{A}_{i j} \frac{d U_{j}}{d t}+\epsilon_{i j k} \Omega_{j} \mathbb{A}_{k l} U_{l}=F_{i}+F_{B i} .
$$

Following a similar procedure, one may obtain Kirchhoff's equation for the angular velocity,

$$
\mathbb{D}_{i j} \frac{d \Omega_{j}}{d t}+\epsilon_{i j k} \Omega_{j} \mathbb{D}_{k l} \Omega_{l}+\epsilon_{i j k} U_{j} \mathbb{A}_{k l} U_{l}=\Gamma_{i} .
$$

The bubble-fixed coordinate system mentioned above is precisely defined as follows. The 1-direction is always parallel to the velocity vector of the bubble. The 2direction is at a right angle to the 1-direction. It is defined such that the 1-2 plane contains both the velocity 


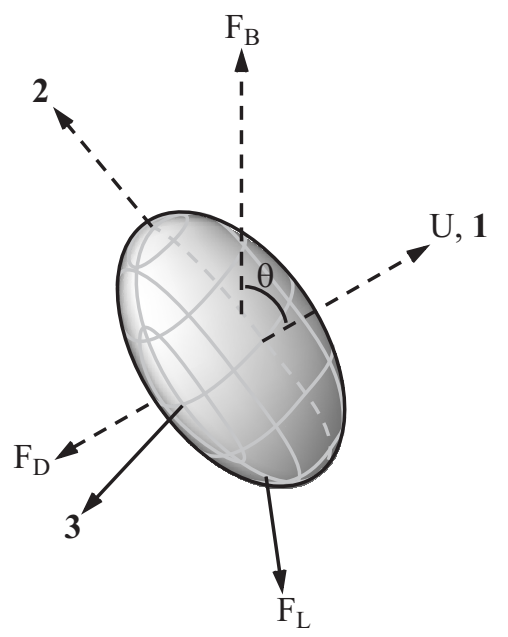

FIG. 5: Diagram of the coordinate system, velocity $U$, pitch angle $\theta$, and external forces $\left(F_{B}, F_{D}, F_{L}\right)$ present for a spiraling bubble. The dashed lines lie in the 1-2 plane.

and the buoyancy force vector and the positive direction coincides with the 2-component of buoyancy. Finally, the 3 -direction is orthogonal to the 1 and 2-directions and, hence, is always purely horizontal. This coordinate system is right-handed and cartesian as illustrated in Fig. 5. With this choice of coordinates, $\mathbf{U}=(U, 0,0), \mathbb{A}$ and $\mathbb{D}$ are diagonal.

\section{B. Hydrodynamic forces and torques}

For an air bubble rising through still water, the forces $\mathbf{F}$, by assumption, include only drag and lift. Drag represents those forces parallel to the bubble trajectory which cannot be accounted for by $F_{B 1}$ and lift represents those forces acting perpendicular to the bubble trajectory which cannot be accounted for by $F_{B 2}$. Generally we have, $\mathbf{F}=\left(F_{D}+F_{B 1}, F_{L 2}+F_{B 2}, F_{L 3}\right)$. History forces are not dealt with explicitly, but rather are implicit in the time dynamics of drag and lift. The torques are assumed to be divided into a rotational $\operatorname{drag} \boldsymbol{\Gamma}_{D}$ and a wake induced torque $\boldsymbol{\Gamma}_{W}$. With these definitions of forces, torques, and coordinate system, the equations 7 and 8 reduce to

$$
\begin{aligned}
\mathbb{A}_{11} \frac{d U}{d t} & =F_{D}+F_{B 1}, \\
\Omega_{3} \mathbb{A}_{11} U & =F_{L 2}+F_{B 2}, \\
-\Omega_{2} \mathbb{A}_{11} U & =F_{L 3} \\
\mathbb{D}_{11} \frac{d \Omega_{1}}{d t} & =\Gamma_{W 1}+\Gamma_{D 1}, \\
\mathbb{D}_{22} \frac{d \Omega_{2}}{d t} & =\Gamma_{W 2}+\Gamma_{D 2}, \\
\mathbb{D}_{33} \frac{d \Omega_{3}}{d t} & =\Gamma_{W 3}+\Gamma_{D 3} .
\end{aligned}
$$

\section{Straight rising bubble equation}

For the size range of bubbles we study, it is has been observed in experiments and numerics that the short axis of the ellipsoidal bubble is always aligned with the bubble velocity vector $[8,10,23]$. A straight rising bubble therefore has $\Omega=0$. After a short initial acceleration from rest, the velocity becomes steady resulting in just one simple equation to describe the motion, namely $F_{D}=-F_{B 1}$. The buoyancy $F_{B 1}=\rho V g$ and drag is conventionally of the form $F_{D}=-0.5 C_{D} \pi R^{2} \rho U^{2}$. For millimeter sized straight rising bubbles, experimental measurements of the drag coefficient $C_{D}$ are well predicted using Moore's theory [18]. Moore's result is

$$
C_{D}=\frac{48}{R e} G(\chi)+\frac{48}{R e^{3 / 2}} G(\chi) H(\chi) .
$$

The first term on the right, $48 G(\chi) /$ Re results from computing the dissipation in the flow field predicted by potential flow theory for an ellipsoid with a free-slip boundary. The second term on the right refines the calculation accounting for the rotational flow in thin boundary layers and a long thin wake. Note that Moore's prediction of $\chi(R)$ does not agree with experiments [9] and therefore one must obtain the aspect ratio empirically.

As shown in figure 3 our bubbles often exhibit a short period of straight rise before the path becomes oscillatory. In section IV, we will use our measurements of straight rise velocity to calculate $C_{D}$ and compare to Moore's prediction.

\section{Zigzagging bubble equation}

For a zigzagging bubble, the angular velocity is just the time derivative of the path pitch angle $\theta$ and is always in the 3 direction, $\boldsymbol{\Omega}=(0,0, \dot{\theta})$. The velocity is unsteady and motion is confined to the 1-2 plane. The resulting equations of motion are

$$
\begin{aligned}
\mathbb{A}_{11} \frac{d U}{d t} & =F_{B 1}+F_{D}, \\
\mathbb{A}_{11} \frac{d \theta}{d t} U & =F_{B 2}+F_{L 2}, \\
\mathbb{D}_{33} \frac{d \theta}{d t} & =\Gamma_{D 3}+\Gamma_{W 3},
\end{aligned}
$$

where the components of the buoyancy force are determined by our path pitch angle measurements, e.g. $F_{B 1}=F_{B} \sin \theta$. The remaining forces and torques are unknown a priori. The drag is no longer expected to match Moore's theory since Moore's calculation was for a closed wake and steady straight line motion. Neither condition holds for a zigzagging bubble. Similarly, no prediction exists for the 2-component of lift $F_{L 2}$, nor for the torques $\Gamma_{D 3}$ and $\Gamma_{W 3}$. However, since we know the trajectory and $\mathbf{F}_{\mathbf{B}}$, we may calculate $F_{D}$ and $F_{L 2}$. These results are presented in section $\mathrm{V}$. 


\section{E. Spiraling bubble equation}

From experimental observations we know that the speed and pitch angle of a spiralling bubble are constant. With path oscillation frequency $f$, one may determine the angular velocity of a spiraling bubble to be $\boldsymbol{\Omega}=(2 \pi f \cos \theta, 2 \pi f \sin \theta, 0)$. The resulting equations of motion are

$$
\begin{aligned}
0 & =F_{B 1}+F_{D}, \\
0 & =F_{b 2}+F_{L 2}, \\
\mathbb{A}_{11} 2 \pi f U \sin \theta & =F_{L 3}, \\
0 & =\Gamma_{D 1}+\Gamma_{W 1}, \\
0 & =\Gamma_{D 2}+\Gamma_{W 2} .
\end{aligned}
$$

As in the zigzag case, the only known force is buoyancy, which leaves lift and drag to be calculated from our measurements. These results are presented in section VI. We remind the reader that for all calculations of forces we account for the increasing volume and aspect ratio $\chi$ caused by the hydrostatic pressure gradient.

Although little is known about $\Gamma_{D}$ and $\Gamma_{W}$, we speculate that these quantities have the potential to be useful for predicting the frequency of spiral oscillation. In the same way that the balance between buoyancy and drag sets the terminal velocity of a straight rising bubble, the balance between the wake induced torque $\Gamma_{W}$ and the drag $\Gamma_{D}$ associated with rotation about the bubble's major axis may determine the rotation rate of the bubble. For a spiralling bubble, this rotation rate is directly tied to spiral frequency as mentioned above. With analytical expressions for $\Gamma_{D}$ and $\Gamma_{W}$, one would likely be able to predict the path oscillation frequency.

\section{STRAIGHT RISE AND ONSET OF PATH INSTABILITY}

Let us discuss several observations of the initial moments of the bubble's ascent up to the point where the trajectory becomes unstable. First, we observe an exponential approach to terminal speed. Second, our measurements of terminal speed agree with Moore's theory [18]. Third, as bubble size is increased the bifurcation to path instability is rather abrupt and possibly subcritical.

As shown in Fig. 6 the bubble accelerates to a terminal velocity $U_{o}$ within the first $200 \mathrm{~ms}$ of the rise. The inset in Fig. 6 shows the velocity $U$ subtracted from the terminal velocity $U_{o}$ and plotted on a logarithmic scale. After about $20 \mathrm{~ms}$, the rise is well approximated by an exponential approach to the terminal velocity. The dashed line in the inset of Fig. 6 is the equation,

$$
U=U_{o}\left(1-\mathrm{e}^{-t / \tau}\right)
$$

where the time constant $\tau=25 \mathrm{~ms}$. We interpret $\tau$ as the approximate time required for the flow around the

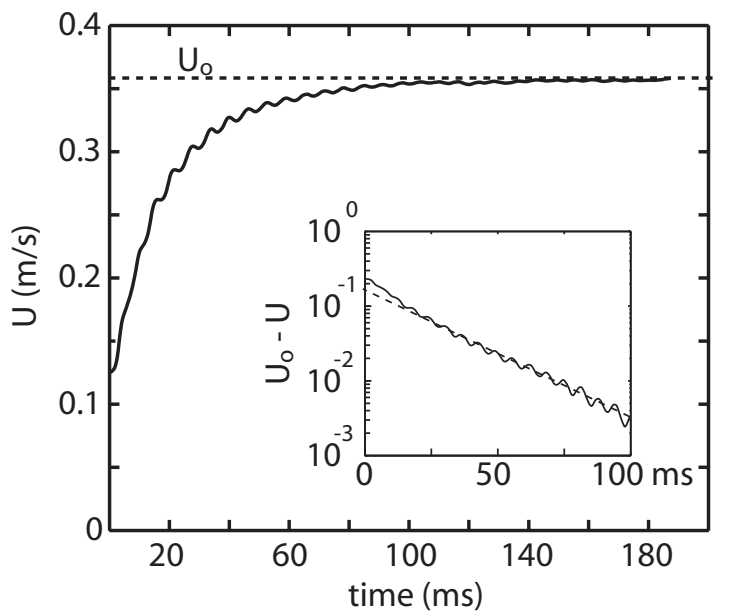

FIG. 6: Velocity during the initial $200 \mathrm{~ms}$ of a bubble's rise. The inset demonstrates the exponential approach to the terminal speed with a time constant of $25 \mathrm{~ms}$. The bubble radius is $1.09 \mathrm{~mm}$ at $1 \mathrm{~atm}$.

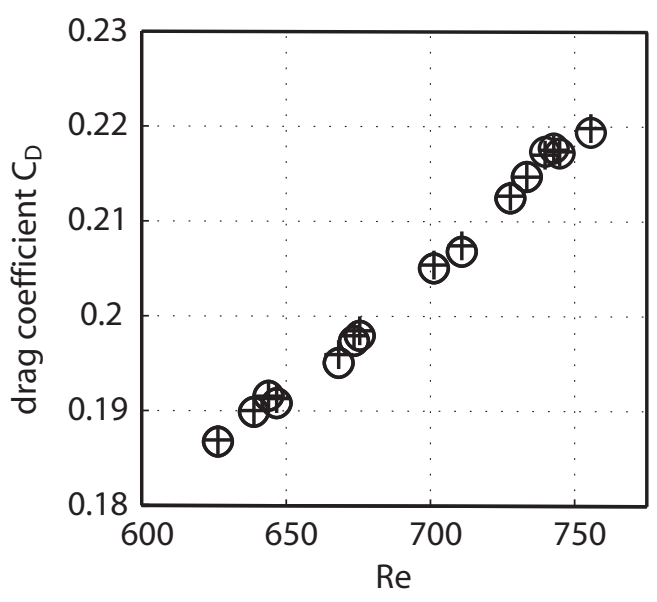

FIG. 7: Comparison of our drag coefficient measurements (circles) during the rectilinear part of the bubble trajectories to predictions of Moore's theory (+).

bubble to respond to a sudden change in the bubble's speed. This time scale will be invoked again in the next section's discussion of zigzag dynamics.

Once the bubble has attained terminal velocity it typically rises for a short period in a straight trajectory before beginning to zigzag. During this constant speed, rectilinear portion of the ascent our velocity measurements are in agreement with Moore's (1965) theory. In Fig. 7, we compare our measurements of the drag coefficient $C_{D}$ as a function of bubble Reynolds number to Moore's prediction (equation 15). The excellent agreement with Moore's theory and, hence, other experiments provides additional validation of our measurement techniques and methods of analysis.

We observe that the height above the release point at which a bubble's path becomes unstable varies signifi- 


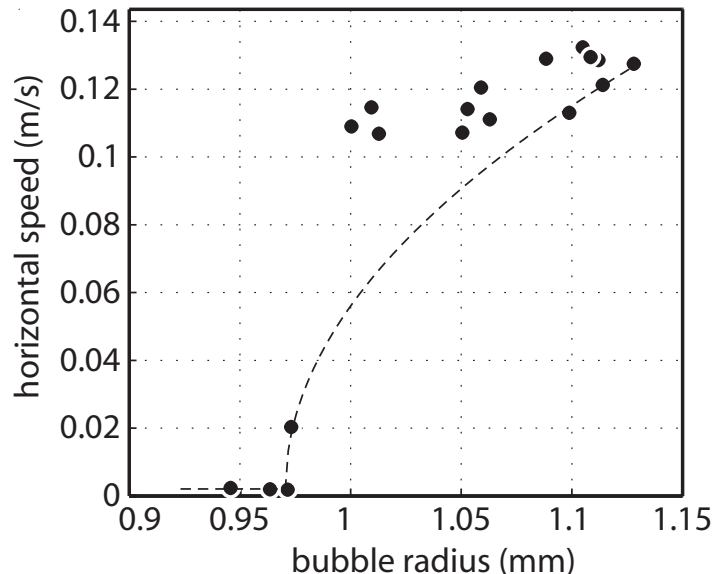

FIG. 8: Horizontal speed of bubble averaged through the interval 1.4 - 1.6 meters above release point. A supercritical bifurcation would correspond to a $\sqrt{R-R_{\text {crit }}}$ behavior (dashed line).

cantly with bubble size. Small bubbles can rise straight for nearly 2 meters before becoming unstable, while larger bubbles may become unstable even before reaching terminal velocity. For those bubbles whose path becomes unstable some time after reaching terminal velocity, we determine the critical radius at the onset of oscillations is $0.97 \mathrm{~mm}$. Using the approximation in eqn. 1, this corresponds to a critical aspect ratio of 2.02. As a measure of the character of the bifurcation from straight to oscillating path, the average horizontal component of velocity between a height of 1.4 and 1.6 meters is shown for a range of bubble sizes in Fig. 8. The transition is rather abrupt as bubble size is increased. This observation suggests the bifurcation to path instability may be subcritical (see comparison to supercritical bifurcation curve $\sqrt{R-R_{\text {crit }}}$ in Fig. 8). Mougin and Magnaudet [23] also suggest that the onset of zigzag motions may be subcritical for increasing aspect ratio. Perhaps one could check for hysteresis experimentally by carefully increasing the hydrostatic pressure (shrinking the bubble size) on an already oscillating bubble.

\section{ZIGZAG FORCE MEASUREMENTS}

As demonstrated in figure 10a the zigzag path is a smooth sinusoid confined to one vertical plane. One important observation is that the speed of the bubble oscillates during the zigzag motion. The speed oscillations are twice the frequency of the path oscillations. The drag and lift forces also oscillate at twice the path oscillation frequency.

First, let us discuss the lift $F_{L 2}$ (see figure 10a). We observe that $\left|F_{L 2}\right|$ reaches a maximum 25-30 ms after the maximum in bubble speed. This lag may be related to the response time $\tau$ reported in the last section. The minimum in $\left|F_{L 2}\right|$ occurs about 25-30 ms after the min- imum bubble speed, again suggesting the importance of the response time $\tau$. Note that $\left|F_{L 2}\right|$ is not zero at the point of inflection of the path as has been suggested by other authors, rather, it is about $25 \mathrm{~ms}$ later. The buoyant force begins to accelerate the bubble again during the moments just before and after the instant when $F_{L 2}=0$. While $F_{L 2}$ is positive the lift is aiding buoyancy to bend the path of the bubble. The sharp drop where $F_{L 2}$ becomes negative again marks the extreme points of the zigzag path where the positive 2 direction reverses by definition of our coordinate system.

We turn now to drag. We observe that the oscillations in $F_{B 1}$ alone cannot account for the oscillations in speed of the zigzagging bubble. Therefore $F_{D}$ must oscillate as well. Remarkably, the oscillations in $F_{D}$ are not as one might expect from standard drag formulas. That is, increasing speed does not coincide with an increase in $\left|F_{D}\right|$. Rather, increasing $\left|F_{D}\right|$ is tied to increasing $\left|F_{L 2}\right|$ as is evident in figure 10a. Thus, the repeating decrease in bubble speed during the zigzag can then be attributed to both a reduction in $F_{B 1}$ as well as an increase in $\left|F_{D}\right|$.

As mentioned in section III, our measurements depend on the assumption of steady bubble shape. While Ellingsen and Risso [10] report steady shape, de Vries et al. [8] suggest that the shape of zigzagging bubbles oscillates slightly. Based on de Vries' schlieren photos, we estimate an upper limit for changes in $\chi$ to be about $10 \%$. Such a variation would result in $5 \%$ changes in the magnitude of $F_{L 2}$ and no more than $1 \mathrm{~ms}$ changes in time dynamics. Therefore, the above discussion would be largely unaffected by such shape changes. For spiralling bubbles de Vries agrees that the shape is steady.

\section{SPIRAL MOTION}

We now turn to the dynamics of spiraling bubble motion. The transition to spiral motion is remarkable in several ways. First, we observe that every zigzagging path eventually becomes a spiral. The spiral may be clockwise or or counterclockwise. Bubbles may zigzag for as many as 15 and as few as 2 cycles before transitioning to the spiral. The transition to spiraling motion is not abrupt, generally developing gradually over several periods of motion as demonstrated above in Fig. 4. Furthermore, the transition does not seem to behave systematically with bubble size. The frequency of path oscillations remains unchanged compared to the zigzag. This is apparent in the horizontal position data shown previously in Fig. 3. The frequency increases as bubble size is increased as shown in Fig. 11a.

The most striking change when the bubble stops zigzagging and begins to spiral is that all the forces and the bubble speed become steady. Fig. 10b shows time series of several features of a spiralling bubble. The top frame presents the component of buoyancy $F_{B 1}$. Since the speed of the bubble is constant during the spiral, $F_{B 1}$ is equal in magnitude to the drag on the bubble. 


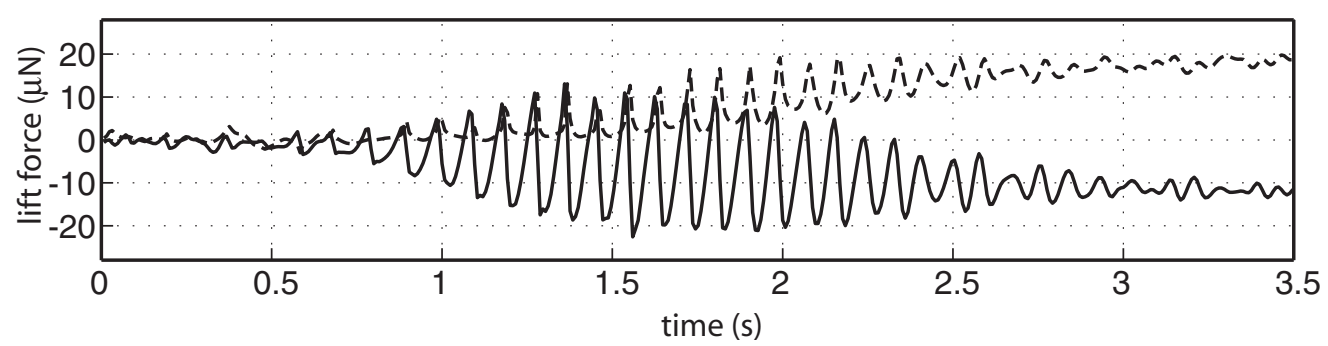

FIG. 9: The two components of lift $\left(F_{L 3}\right.$-dotted line, $F_{L 2^{-}}$solid line) and as measured during the trajectory shown in Fig. 3. The bubble radius is $1.12 \mathrm{~mm}$ at $1 \mathrm{~atm}$. The measurement uncertainty is about $\pm 4 \mu \mathrm{N}$.

(a) zigzag

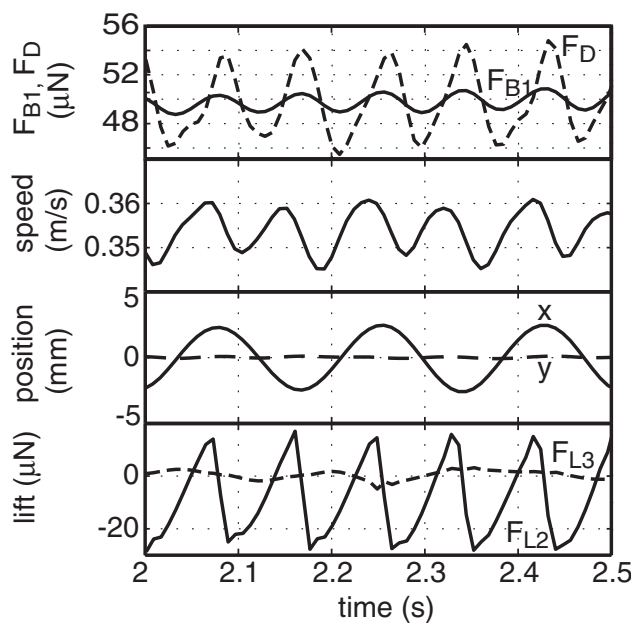

(b) spiral

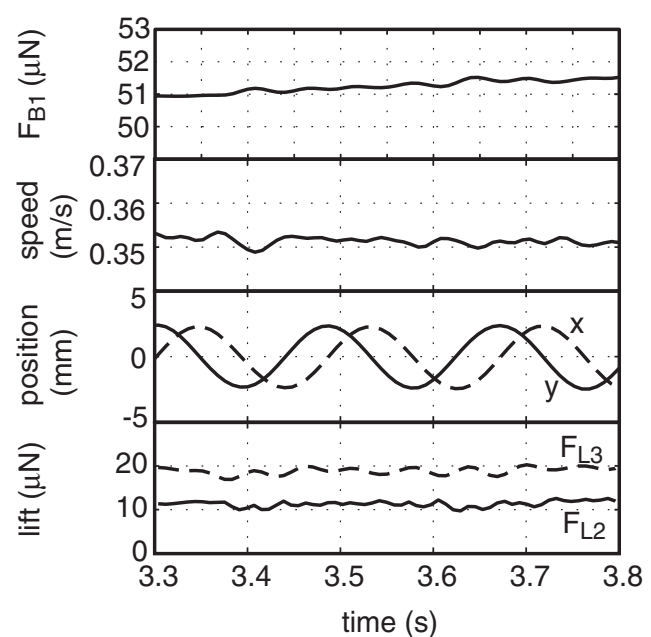

FIG. 10: The buoyancy component tangential to the path $F_{B 1}$ (solid line), drag $F_{D}$ (dashed line), bubble velocity, horizontal position ( $\mathrm{x}$ and $\mathrm{y}$ ), and lift force (solid line: $F_{L 2}$ and dashed line: $F_{L 3}$ ) as measured during a zigzagging trajectory (a) and a spiraling trajectory (b). The bubble radius is $1.12 \mathrm{~mm}$ at $1 \mathrm{~atm}$. The measurement uncertainties are $\pm 0.5 \mu \mathrm{N}$ for $F_{B 1}$ and $F_{D}$, $\pm 7 \mathrm{~mm} / \mathrm{s}$ for speed, $\pm 0.2 \mathrm{~mm}$ for position, and $\pm 4 \mu \mathrm{N}$ for lift forces.

We observe the magnitude of this drag is very nearly equal to that predicted for a bubble at the same speed using Moore's formula. This observation is surprising since Moore's theory is based on different flow around the bubble and the drag during the zigzag is clearly not well described by Moore's theory. The component of lift $F_{L 2}$ is constant in time, balancing $F_{B 2}$. We observe that $F_{L 3}$ is typically about twice as large as $F_{L 2}$, and also constant in time. This is apparent in Figs. 9 and 10b and is quantified for a range of bubble sizes in Fig. 11b.

\section{CONCLUSIONS}

We make precise three-dimensional measurements of trajectories and speed of millimeter sized air bubbles rising through $2 \mathrm{~m}$ of still water. We use these measurements to calculate drag and lift forces acting on the bubble.

We observe that for the rectilinear portion of bubble trajectories the measured drag matches Moore's prediction. The bifurcation to path instability is abrupt and perhaps subcritical. The bifurcated state always begins as a zigzag and evolves into a spiral. We measure 10 $\mu \mathrm{N}$ oscillations in drag for a zigzagging bubble and lift forces on both zigzagging and spiraling bubbles $10-40 \mu \mathrm{N}$ in magnitude (buoyancy is typically $50-60 \mu \mathrm{N}$ ).

\section{ACKNOWLEDGEMENTS}

This paper benefitted from discussions at the Euromech colloquium 465, "Hydrodynamics of bubbly flows". We thank Jacques Magnaudet for helpful advice on Kirchhoff equations. The skilled work of Denis Le Tourneau and Pascal Metz in building the water tank and the ultrasound device respectively is also appreciated. This work was funded by École Normale Supérieure, Centre National de la Recherche Scientifique, and Région Rhône-Alpes, Emergence Contract 0501551301. 

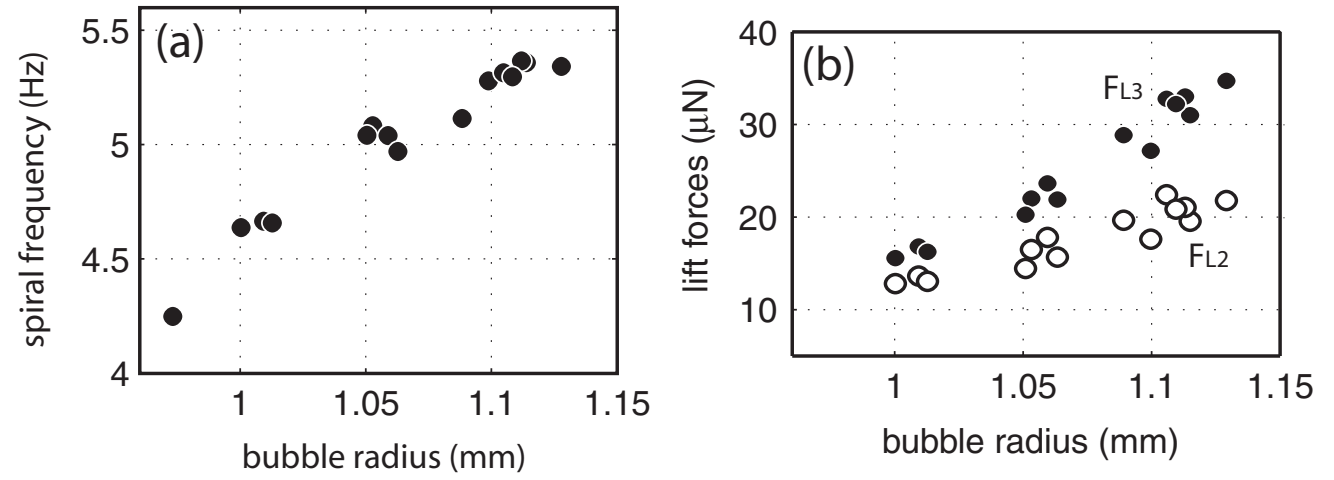

FIG. 11: (a) The frequency of spiraling motion for a range of bubble sizes. The measurement is precise to within $2 \%$. (b) Comparison of $F_{L 2}$ (open circles) and $F_{L 3}$ (solid circles) for spiraling bubbles of various sizes. The measurement uncertainty is $\pm 2 \mu \mathrm{N}$.

[1] N. M. Aybers and A. Tapucu, "The motion of gas bubbles rising through stagnant liquid," Wärme- und Stoffübertragung 2, 118 (1969).

[2] N. M. Aybers and A. Tapucu, "Studies on the drag and shape of gas bubbles rising through stagnant liquid," Wärme- und Stoffübertragung 2, 171 (1969).

[3] Batchelor, G. K., An introduction to fluid dynamics (Cambridge University Press, Cambridge, 1967).

[4] T. B. Benjamin, "Hamiltonian theory for motions of bubbles in an infinite liquid," J. Fluid Mech. 181, 349 (1984).

[5] C. Brücker, "Structure and dynamics of the wake of bubbles and its relevance for bubble interaction," Phys. Fluids 11, 1781 (1999).

[6] R. Clift, J. R. Grace, and M. E. Weber, "Bubbles, Drops, and Particles (Academic, New York, 1978).

[7] A. W. G. de Vries, "Path and wake of a rising bubble," (PhD dissertation, University of Twente, Netherlands, 2001).

[8] A. W. G. de Vries, A. Biesheuvel, and L. van Wijngaarden, "Notes on the path and wake of a gass bubble rising in pure water," Int. J. Multiphase Flow 28, 1823 (2002).

[9] P. C. Duineveld, "The rise velocity and shape of bubbles in pure water at high Reynolds number," J. Fluid Mech. 292, 325 (1995).

[10] K. Ellingsen and F. Risso, "On the rise of an ellipsoidal bubble in water: oscillatory paths and liquid-induced velocity," J. Fluid Mech. 440, 235 (2001).

[11] R. A. Hartunian and W. R. Sears, "On the instability of small gas bubbles moving uniformly in various liquids," J. Fluid Mech. 3, 27 (1957).

[12] M. S. Howe, "On the force and moment on a body in an incompressible fluid, with application to rigid bodies and bubbles at low and high Reynolds numbers", Q. J. Mech. Appl. Math. 48, 401 (1995).

[13] H. Lamb, Hydrodynamics, 6th ed. (Dover Publications, New York, 1945).

[14] L. G. Leal, "Vorticity transport and wake structure for bluff bodies at finite Reynolds number," Phys. Fluids A 1, 124 (1989).

[15] K. Lunde and R. J. Perkins, "Observations on wakes behind speroidal bubbles and particles," Paper No.
FEDSM'97-3530, 1997 ASMEFED Summer Meeting, Vancouver, Canada, page 1.

[16] J. Magnaudet and I. Eames, "The motion of highReynolds-number bubbles in inhomogeneous flows," Ann. Rev. Fluid Mech. 32, 659 (2000).

[17] J. Mercier, A. Lyrio, and R. Forslund, "Three dimensional study of the nonrectilinear trajectory of air bubbles rising in water," J. App. Mech. 40, 650 (1973).

[18] D. W. Moore, "The velocity of rise of distorted gas bubbles in a liquid of small viscosity," J. Fluid Mech. 23, 749 (1965).

[19] N. Mordant, J.-F. Pinton, and O. Michel, "Time-resolved tracking of a sound scatterer in a complex flow: Nonstationary signal analysis and applications," J. Acoust. Soc. Am. 112, 108 (2002).

[20] N. Mordant and J.-F. Pinton, "Velocity measurements of a settling sphere," Eur. Phys. J. B 18, 343 (2000).

[21] N. Mordant, P. Metz, O. Michel, and J.-F. Pinton, "An acoustic technique for Lagrangian velocity measurements," Rev. Sci. Instr. 76, 025105 (2005).

[22] G. Mougin and J. Magnaudet, "The generalized Kirchhoff equations and their application to the interaction between a rigid body and an arbitrary time-dependent viscous flow", Int. J. Mulitphase Flow 28, 1837 (2002).

[23] G. Mougin and J. Magnaudet, "Path instability of a rising bubble." Phys. Rev. Lett. 88, 014502 (2002).

[24] G. Mougin, "Interactions entre la dynamique d'une bulle et les instabilitiés de son sillage," ( PhD Dissertation, Institute National Polytechnique de Toulouse, France, 2002).

[25] C. D. Ohl, A. Tijink and A. Prosperetti, "The added mass of an expanding bubble," J. Fluid Mech. 482, 271 (2003).

[26] A. Prosperetti, "Bubbles," Phys. Fluids 16, 1852 (2004).

[27] P. G. Saffman, "On the rise of small air bubbles in water," J. Fluid Mech. 1, 249 (1956).

[28] H. Sakamoto and H. Hanui, "The formation mechanism and shedding frequency of vortices from a sphere in uniform shear flow," J. Fluid Mech. 287, 151 (1995).

[29] C. Veldhuis, Personal communications, 2005.

[30] M. Wu and M. Gharib, "Experimental studies on the shape and path of small air bubbles rising in clean water," 
Phys. Fluids 14, L49 (2002). 